\title{
Network analysis of the effects of long non-coding RNAs in artemisinin treatment of atherosclerosis in $\mathrm{APOE}^{-/-}$mice
}

\author{
Zhao Qiao, Du Hongjiao, Li Xiaodong
}

Department of Cardiology, Shenjing Hospital of China Medical University, Shenyang, Liaoning, China

Submitted: 9 August 2019; Accepted: 22 February 2020

Online publication: 28 March 2021

Arch Med Sci

DOI: https://doi.org/10.5114/aoms/118378

Copyright $\odot 2021$ Termedia \& Banach

\section{Abstract}

Introduction: Atherosclerosis has become a worldwide medical burden. Our previous studies have shown that artemisinin (ART) had the capability to reduce atherosclerosis. Emerging evidence indicates that long non-coding RNAs (IncRNAs) are involved in the development of atherosclerosis. However, whether IncRNAs might participate in the mechanism through which artemisinin mitigates atherosclerosis has not been reported.

Material and methods: Eight-week-old apolipoprotein E deficient $\left(\mathrm{APOE}^{-/-}\right)$ mice were divided into two groups, one of which was treated with artemisinin. Red oil O staining was used to measure the sizes of the atherosclerotic lesions. We conducted deep sequencing to investigate IncRNA profiles in the aorta tissue in high-fat diet fed APOE knockdown mice with and without artemisinin treatment. CeRNA network, Kyoto Encyclopedia of Genes and Genomes (KEGG) and Gene Ontology (GO) analyses were performed through bioinformatics analysis. RT-PCR was used to validate the differentially expressed IncRNAs.

Results: A total of 102 IncRNAs and 4,630 mRNAs were differentially expressed $(p<0.05)$ between the artemisinin treatment group and atherosclerosis model group. KEGG and GO analyses indicated that the categories metabolic process, specific amino acid degradation and PI3K-Akt signaling pathway are involved in the effects of artemisinin treatment in atherosclerosis ( $q$ < 0.05). LncRNA ENSMUST00000099676.4, ENSMUST00000143673.1, ENSMUST00000070085.5 and ENSMUST00000224554 might be engaged in the treatment mechanism through which artemisinin alleviates atherosclerosis. Conclusions: These findings indicated the possible mechanism and therapeutic role of IncRNAs in artemisinin treatment of atherosclerosis and provided a theoretical basis for the future application of artemisinin in patients with atherosclerosis.

Key words: Artemisia annua, therapeutics, coronary disease, inflammation.

\section{Introduction}

Atherosclerosis, a chronic progressive disease, is characterized by fibrous and lipid plaques that narrow and harden arteries, thus resulting in various diseases such as coronary heart disease, peripheral artery disease and stroke. Two main factors, lipids and inflammation, are considered to be involved in the formation of atheromas [1-3]. In the past, the main strategies for treating atherosclerosis focused on regulating lipid metabolism and

\author{
Corresponding author: \\ Li Xiaodong MD \\ Department of Cardiology \\ Shenjing Hospital of China \\ Medical University \\ No.36, Sanhao Street, \\ Heping District \\ Shenyang 110004 , \\ Liaoning, China \\ E-mail: licardio@163.com
}


lowering low-density lipoprotein. Recently, some anti-inflammatory drugs began to be used to treat atherosclerosis [4-6]. Nevertheless, the choice of antiinflammatory drugs is very limited. For instance, some non-steroidal anti-inflammatory drugs may even increase the risk of cardiovascular events [4]. Therefore, finding new anti-atherosclerotic drugs and determining their mechanisms are crucial.

Artemisinin (ART), which was first extracted from Artemisia annua by Tu Youyou, has been used as an efficient drug for curing malaria [7]. In addition to its anti-malarial activity, ART can be used to treat other diseases such as neuroinflammation, cancer and lupus erythematosus, owing to its outstanding anti-inflammatory and anti-angiogenetic characteristics [8, 9]. Hence, we hypothesized that ART might alleviate atherosclerosis. We were excited to find that ART inhibited the proliferation of vascular smooth muscle cells (VSMCs) in vitro and alleviated atherosclerotic plaque formation in vivo [10-12]. Nonetheless, ART is not clinically used to treat atherosclerosis because the mechanism of ART against atherosclerosis was unclear.

Long non-coding RNAs (IncRNAs) are linear RNAs that do not encode proteins and used to be considered junk RNA. In recent years, researchers have found that numerous IncRNAs are involved in the regulation of the cell cycle, proliferation and apoptosis [13, 14]. Herein, we hypothesized that IncRNAs are engaged in the effects of ART on atherosclerosis. Through high-throughput sequencing techniques, we detected the differentially expressed IncRNA profiles in an atherosclerotic model mice treated with ART (ART group) and atherosclerosis model mice without ART treatment (AS group), and explored the possible mechanism through which ART alleviates atherosclerosis in mammals. This study aims to provide a theoretical basis for the future use of artemisinin in the treatment of atherosclerosis.

\section{Material and methods}

\section{Ethical statement}

This study was approved by the Animal Ethics Committee of Shengjing Hospital (Ethic No. 2018PS513K). Animal experiments followed the guidelines of the European Convention for the Protection of Vertebrate Animals used for Experimental and Other Scientific Purposes and the Animal Research: Reporting of In Vivo Experiments guidelines.

\section{Groups, atherosclerosis modeling and ART treatment}

Eight-week-old male C57BL6 mice and apolipoprotein $\mathrm{E}$ deficient $\left(\mathrm{APOE}^{-/-}\right)$mice with the same genetic background were purchased from Beijing Huafukang Biotechnology Co., Ltd. (Beijing, PR China).
Eight C57BL/6 mice fed a standard rodent chow diet served as the control group. Sixteen $\mathrm{APOE}^{-/-}$mice were randomly divided into two groups, eight in the atherosclerosis group and eight in the ART group. Mice in the atherosclerosis group were given a Western diet (21\% fat, 0.15 cholesterol, Beijing Huafukang Biotechnology, No. H10141). Mice in the ART group were given a Western diet and were administered ART (purity > 98\%, Beijing Solarbio Science \& Technology Co., Ltd., IA0520) orally at $50 \mathrm{mg} / \mathrm{kg}$ / day as described previously [15]. The three groups were housed in a temperate room at a temperature of $22 \pm 1^{\circ} \mathrm{C}$ and humidity of $45-55 \%$. The treatment process described above lasted 8 weeks.

\section{Specimen acquisition}

Specimen acquisition was performed when the mice were 16 weeks old. All mice were euthanized through intraperitoneal injection of pentobarbital. The hearts of the mice were separated and fixed in $4 \%$ paraformaldehyde for Oil Red 0 staining. The intact aortas of the mice of the atherosclerosis group and ART group were carefully separated from the surrounding adherent tissue and stored in liquid nitrogen for the bioinformatics analysis.

\section{Oil Red O staining}

To visualize the atherosclerotic lipid accumulation, we stained the heart slices of the mice with Oil Red O (Sigma-Aldrich O-0625). In brief, the heart was cut into sections perpendicular to the long axis of the heart, and the aortic valve plane was chosen to perform Oil Red O staining. After rinsing with phosphate buffered saline, the aortic valve slices were stained with Oil Red O for 25 min at room temperature. Then the slices were rinsed with $60 \%$ isopropyl alcohol and distilled water. Ultimately, the images of Oil Red O staining aortic valve slices were obtained with a microscope (Nikon Eclipse NI, Japan). Positively stained areas of five sections from each mouse were quantified through Image by a researcher who was blind to the experimental condition and the results were presented as the mean lesion area.

\section{RNA extraction and quality control}

Total RNA was extracted from the aortas of the mice with RNAiso Plus (Takara Bio Inc., Japan) in accordance with the protocol. The quality and quantity of RNA were assessed with a NanoDrop ND-1000 spectrophotometer. The OD260/280 ratios of all samples were between 1.8 and 2 .

\section{High-throughput sequencing}

The IncRNA and mRNA libraries were generated from the total RNAs extracted from aorta samples of three randomly selected mice in the atheroscle- 
rosis group and ART group with an rRNA-depleted Preparation Kit for Illumina (Neb, USA), according to the protocol. Then the libraries were sequenced on the Illumina HiSeq 4000 platform according to the vendor's instructions by NovoGene Genome Sequencing Company (Beijing, China).

\section{The ceRNA network construction}

LncRNA-miRNA networks were constructed in StarBase v3.0. Then we used StarBase v3.0 and TargetScan $v 7.2$ to predict the miRNA-mRNA interactions. LncRNA-miRNA-mRNA networks conforming to the competing endogenous RNAs (ceRNA) rules were depicted in Cytoscape v3.7.1.

\section{KEGG and GO analysis}

Gene Ontology (http://www.geneontology.org) analysis of biological processes, cellular components and molecular functions was used to classify the functions of differentially expressed mRNAs. Kyoto Encyclopedia of Genes and Genomes (http://www.genome.jp/kegg) pathway analysis was used to indicate the main biochemical and signaling pathways in which differentially expressed genes participate.

\section{Quantitative real-time polymerase chain reaction}

We selected RNA samples from 5 mice per group for validation. cDNA was synthesized by using the PrimeScript RT with gDNA Eraser reagent kit (Takara, RR047A). Quantitative real-time polymerase chain reaction (qRT-PCR) was performed with $2 \mu \mathrm{l}$ of cDNA, TB Green Premix Ex Taq II (Takara, RR820A), and gene specific primers which were synthesized by Sangon Biotech (Shanghai, China) (Table I). $\beta$-actin was used as a reference. The specificity was confirmed on the basis of the single peak on the melting curve. Three individual technical replicates were examined for the sample. The relative levels normalized to beta-actin were calculated with the $2^{-\Delta \Delta C T}$ method.

\section{Statistical analysis}

The statistical data were analyzed in GraphPad Prism 7 (GraphPad Software, USA). Comparisons between the two groups were analyzed with Student's $t$-test. A value of $p<0.05$ was considered to indicate statistical significance.

\section{Results}

\section{ART alleviated atherosclerosis in $\mathrm{APOE}^{-/-}$mice}

We established three groups: a control group consisting of $\mathrm{C} 57 \mathrm{BL} / 6$ mice, an AS group consisting of high-fat diet fed $\mathrm{APOE}^{-/-}$mice without ART treatment and an ART group consisting of highfat diet fed $\mathrm{APOE}^{-/-}$mice with ART treatment. Oil red $O$ staining was used to evaluate the severity of aortic atherosclerosis. The area of atherosclerotic plaques in the atherosclerosis group (Figure $1 \mathrm{~B} ; 1.77 \pm 0.14 \mathrm{~mm}^{2}$ ) was significantly larger than that in the control group (Figure $1 \mathrm{~A}$; $0.04 \pm 0.02 \mathrm{~mm}^{2} ; p<0.0001$ ), thus confirming the success of atherosclerosis modeling (Figure $1 \mathrm{D})$. Compared with that in the atherosclerosis group, there was a significantly smaller area of atherosclerotic plaques in the ART group (Figure $1 \mathrm{C} ; 0.83 \pm 0.10 \mathrm{~mm}^{2} ; p<0.01$ ). This result illustrated the anti-atherosclerosis effect of ART and laid a foundation for subsequent bioinformatics analyses.

\section{Screening of differentially expressed IncRNAs and mRNAs}

The expression profiles were compared between the main artery samples of the ART group and atherosclerosis group. Among a total of 3625 IncRNAs and 55831 mRNAs detected by high-throughput sequencing, there were significant differences in the expression of 102 IncRNAs and 4630 mRNAs $(p<0.05)$. Furthermore, 22 lnCRNAs decreased and 5 IncRNAs increased in the ART group by showing significantly differential expression with an absolute $\log _{2}$ (fold change) $>1$ ( $p<0.05)$ (Table II). Among these differentially expressed IncRNAs, 50 IncRNAs decreased, and 52 IncRNAs increased in the ART group (Figure $2 \mathrm{~A}$ ). Moreover, compared with the atherosclerosis group, in the ART group, 2595 mRNAs decreased, and 2035 mRNAs increased (Figure 2 B). The top 10 differentially expressed mRNAs are listed in Table III.

Table I. Primers of IncRNAs

\begin{tabular}{|lcc|}
\hline & Forward & Reverse \\
\hline ENSMUST00000099676.4 & AAGTCTTCTGCCATCAAGCCA & CAGTGGGTGGCTTTTTCCAC \\
\hline ENSMUST00000143673.1 & GCCTCGGAGATGAAAGATGGA & CACAAACTAACAGGCAGCCAC \\
\hline ENSMUST00000070085.5 & AAGTTACAGCCACATGCTGAGA & ATCCATCTGGTCAGGGTCAC \\
\hline ENSMUST00000182520.1 & GTCTTGCGCGGTCTTGGA & GGACGAACCCACCGTGAACT \\
\hline ENSMUST00000224554 & AGAAAATTCAGCCACTGCCA & CAGCTCCTTCAAGCCAGTGA \\
\hline ENSMUST00000141700.1 & AGAGGGCATTGCTGTGACAA & GCATGTTGAGGACAGAGCCT \\
\hline
\end{tabular}



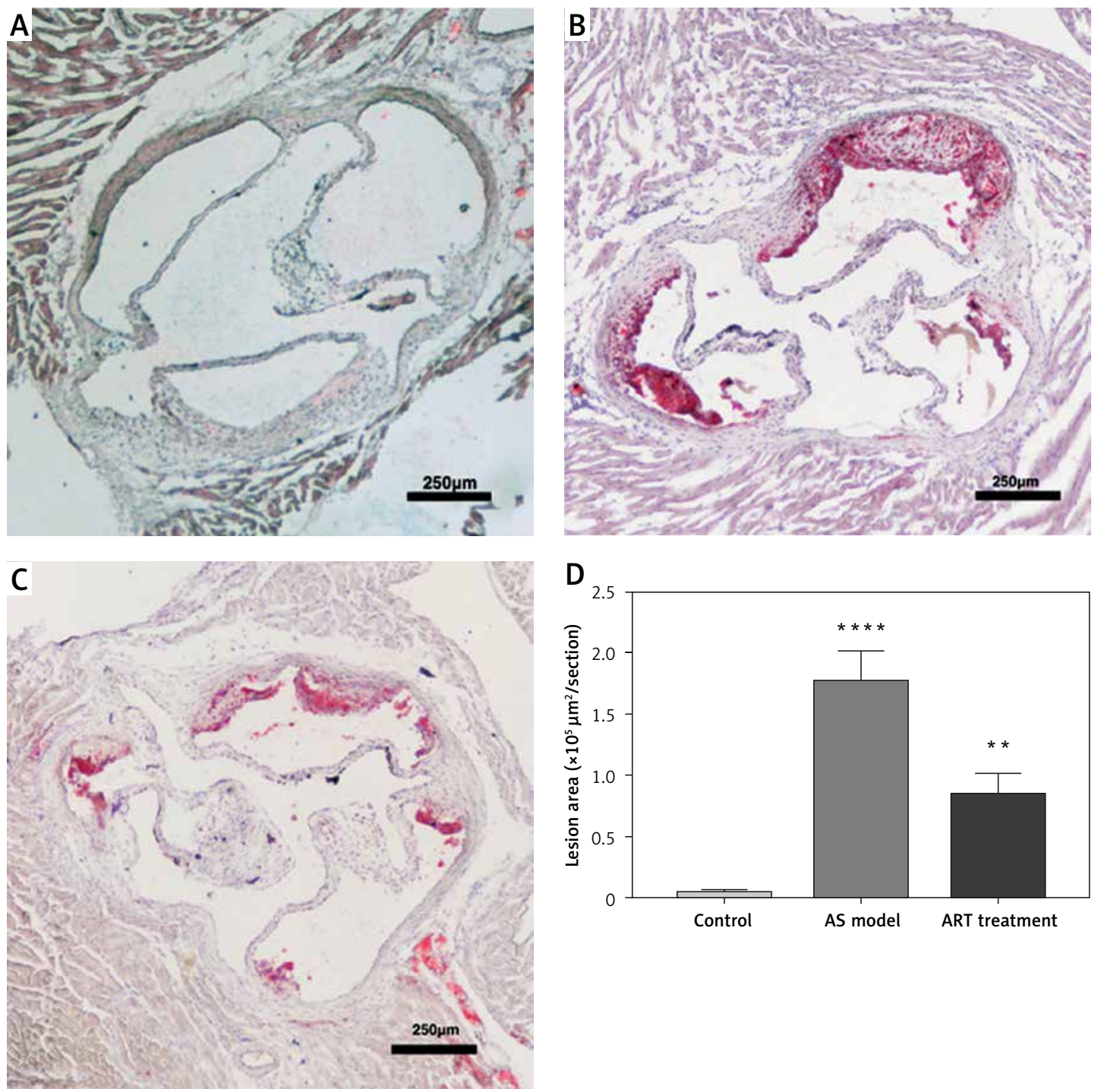

Figure 1. Artemisinin alleviated atherosclerosis. The area of atherosclerotic plaques in the atherosclerosis group (B) was significantly larger $(p<0.0001)$ than that in the control group (A). The area of plaques in the ART group (C) was significantly smaller $(p<0.01)$ than that in the atherosclerosis group. $\mathbf{D}$ - Statistical results

${ }^{* *} p<0.001$.

\section{Predicted networks of ceRNAs}

The networks of ceRNAs were constructed on the basis of increased and decreased IncRNAs. By using StarBase v3.0, among those differentially expressed IncRNAs, 35 upregulated and 22 downregulated IncRNAs can be predicted to have their potential binding miRNAs. Then the predicted miRNAs whose trends were compatible with the high-throughput sequencing data were input into two databases (StarBase v3.0 and TargetScan v7.2) to predict the interaction of miRNAs and mRNAs. In the same way, the predicted mRNAs whose trends were compatible with the high-throughput sequencing data were filtered. Ultimately, with Cytoscape v3.7.1, we determined and depicted the relevance of IncRNAs, miRNAs and mRNAs (Figure 3). Twelve increased miRNAs and four decreased
miRNAs in the ART group were found in the ceRNA network. LncRNA ENSMUST00000099676.4 was predicted to interact with miR-370. LncRNA ENSMUST00000143673.1 and IncRNA ENSMUST00000070085.5 were predicted to interact with let-7a. LncRNA ENSMUST00000224554 was predicted to interact with miR-145. The predicted downstream miRNAs mentioned above have been reported to be associated with AS.

\section{$\mathrm{GO}$ and KEGG analysis}

GO analysis indicated that specific mRNAs involved in biological processes (e.g., metabolic process and biological regulation), cellular components (e.g., organelle and intracellular) and molecular functions (e.g., binding and catalytic activity) were enriched. The KEGG analysis illustrated that 
these differentially expressed mRNAs were associated with atherosclerosis, through categories including amino acid degradation, oxidative phosphorylation, peroxisome, fatty acid metabolism and the CGMP-PKG signaling pathway (Figure 4).

\section{Validation of differentially expressed IncRNAs}

To validate the high-throughput sequencing results, we used qRT-PCR to detect six decreased IncRNAs of interest. Compared with those in the atherosclerosis group, levels of five of six IncRNAs (ENSMUST00000099676.4, ENSMUST00000143673.1, ENSMUST00000070085.5, ENSMUST00000182520.1, and ENSMUST00000224554) were lower in the ART group. However, the expression level of IncRNA ENSMUST00000141700.1 showed no significant difference between the ART group and atherosclerosis group (Figure 5).

\section{Discussion}

Atherosclerosis imposes a worldwide medical disease burden, especially in developed countries [16]. Although anti-lipid drugs and anti-inflammatory drugs are widely used to cope with this severe medical burden, the presence of numerous side effects makes those drugs not suitable for all people. Thus, finding a new treatment with few side effects has become a focus of attention. Recently, ART, an excellent anti-malaria drug with high safety and low side effects in clinic use, has been found to possess anti-atherosclerotic properties. However, the precise mechanism through which ART mitigates atherosclerosis remains unclear. More laboratory evidence is needed to confirm the anti-atherosclerotic capacity of ART, to lay a foundation for future clinical applications. Hence, in this study, by using transcriptome sequencing technology, we analyzed the possible mechanism through which ART alleviates atherosclerosis in mammals.

A

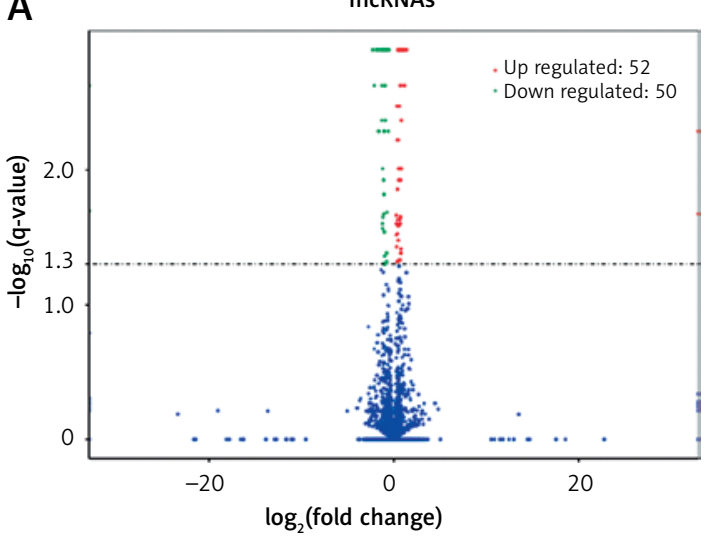

Table II. Differentially expressed IncRNA

\begin{tabular}{|c|c|c|}
\hline Transcript id & $\begin{array}{l}\log 2 \text { (fold- } \\
\text { change) }\end{array}$ & $P$-value \\
\hline LNC_000243 & -2.287 & $<0.0001$ \\
\hline LNC_000658 & -2.10364 & $<0.001$ \\
\hline ENSMUST00000224554 & -1.86235 & $<0.0001$ \\
\hline ENSMUST00000194137.1 & -1.77807 & $<0.0001$ \\
\hline ENSMUST00000099676.4 & -1.65095 & $<0.0001$ \\
\hline LNC_001443 & -1.64415 & $<0.001$ \\
\hline ENSMUST00000195382.1 & -1.44281 & $<0.0001$ \\
\hline ENSMUST00000189288.1 & -1.39193 & $<0.0001$ \\
\hline ENSMUST00000123127.2 & -1.32681 & $<0.0001$ \\
\hline LNC_000640 & -1.30855 & $<0.001$ \\
\hline LNC_000043 & -1.29599 & $<0.001$ \\
\hline ENSMUST00000040608.3 & -1.29442 & $<0.0001$ \\
\hline ENSMUST00000201732.1 & -1.27859 & $<0.0001$ \\
\hline ENSMUST00000145438.1 & -1.23816 & $<0.01$ \\
\hline LNC_001676 & -1.23582 & $<0.0001$ \\
\hline ENSMUST00000202328.1 & -1.22083 & $<0.01$ \\
\hline ENSMUST00000194467.1 & -1.21467 & $<0.001$ \\
\hline ENSMUST00000204370.2 & -1.18268 & $<0.01$ \\
\hline LNC_000959 & -1.14247 & $<0.01$ \\
\hline ENSMUST00000197086.1 & -1.11396 & $<0.0001$ \\
\hline LNC_000285 & -1.10797 & $<0.001$ \\
\hline ENSMUST00000199326.1 & -1.07832 & $<0.01$ \\
\hline LNC_001532 & -1.07202 & $<0.0001$ \\
\hline ENSMUST00000160679.7 & -1.0645 & $<0.0001$ \\
\hline ENSMUST00000204288.1 & -1.05414 & $<0.0001$ \\
\hline ENSMUST00000186147.6 & -1.02893 & $<0.01$ \\
\hline ENSMUST00000186444.1 & -1.01811 & $<0.01$ \\
\hline LNC_000090 & 1.06538 & $<0.0001$ \\
\hline ENSMUST00000221461 & 1.06987 & $<0.0001$ \\
\hline ENSMUST00000133499.1 & 1.169 & $<0.001$ \\
\hline ENSMUST00000180386.1 & 1.19212 & $<0.0001$ \\
\hline ENSMUST00000132056.7 & 1.37521 & $<0.0001$ \\
\hline
\end{tabular}

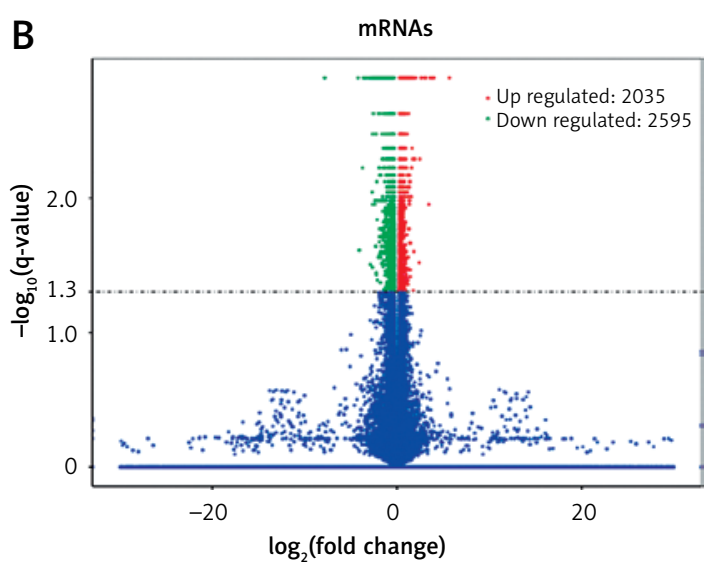

Figure 2. Volcano plot of differentially expressed IncRNAs (A) and mRNAs (B) 
Table III. Top 10 mRNAs

\begin{tabular}{|lccc|}
\hline Transcript id & Gene name & $\log _{2}$ (foldchange) & $P$-value \\
\hline ENSMUST00000032089 & Reg3g & 5.68924 & $<0.0001$ \\
\hline ENSMUST00000076155 & Eno1b & 4.02551 & $<0.0001$ \\
\hline ENSMUST00000165147 & Muc5b & 3.8935 & $<0.0001$ \\
\hline ENSMUST00000035077 & Ltf & 3.64403 & $<0.0001$ \\
\hline ENSMUST00000112022 & Camp & 3.46233 & $<0.001$ \\
\hline ENSMUST00000022692 & Sftpc & -7.82539 & $<0.0001$ \\
\hline ENSMUST00000028233 & Hc & -4.21105 & $<0.0001$ \\
\hline ENSMUST00000032974 & Atp2a1 & -4.07453 & $<0.01$ \\
\hline ENSMUST00000103095 & Tnnc2 & -3.71702 & $<0.001$ \\
\hline ENSMUST00000065588 & Gbp10 & -3.66993 & $<0.0001$ \\
\hline
\end{tabular}

Over the past decade, IncRNAs have been found to be involved in the regulation of critical physiological and pathological processes in cells via diverse mechanisms [13]. Recent studies have indicated that IncRNAs participate in atherosclerosis [14]. The IncRNA H19 is upregulated in the plasma of patients with atherosclerosis and regulates ox-LDL-induced endothelial cell injury by acting as a let-7a sponge [17]. Interestingly, in our study, IncRNA H19 was decreased, and let-7a was increased in the ART treatment group, thus indicating one possible treatment mechanism through which ART decreases atherosclerosis.

CeRNAs are a group of RNAs that regulate transcripts of other RNAs by competing for shared miRNAs. LncRNAs regulate the expression of target genes by serving as miRNA sponges via ceRNA networks. Thus, we predicted the ceRNA networks of decreased and increased IncRNAs. Most of the increased miRNAs predicted by the downregulat-

A

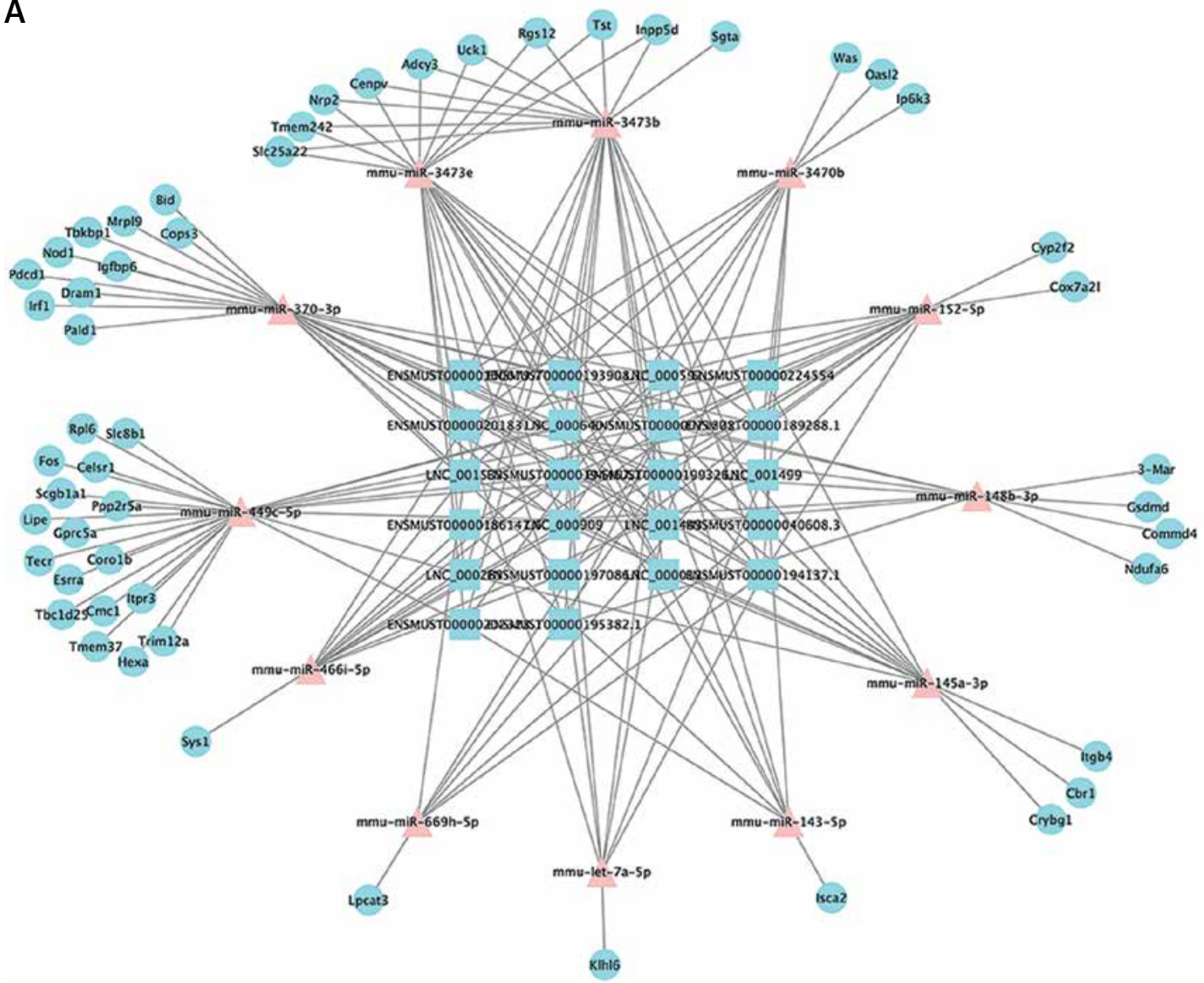

Figure 3. Predicted networks of ceRNAs. The ceRNA networks of decreased IncRNAs (A) and increased IncRNAs (B) in the ART group are depicted 


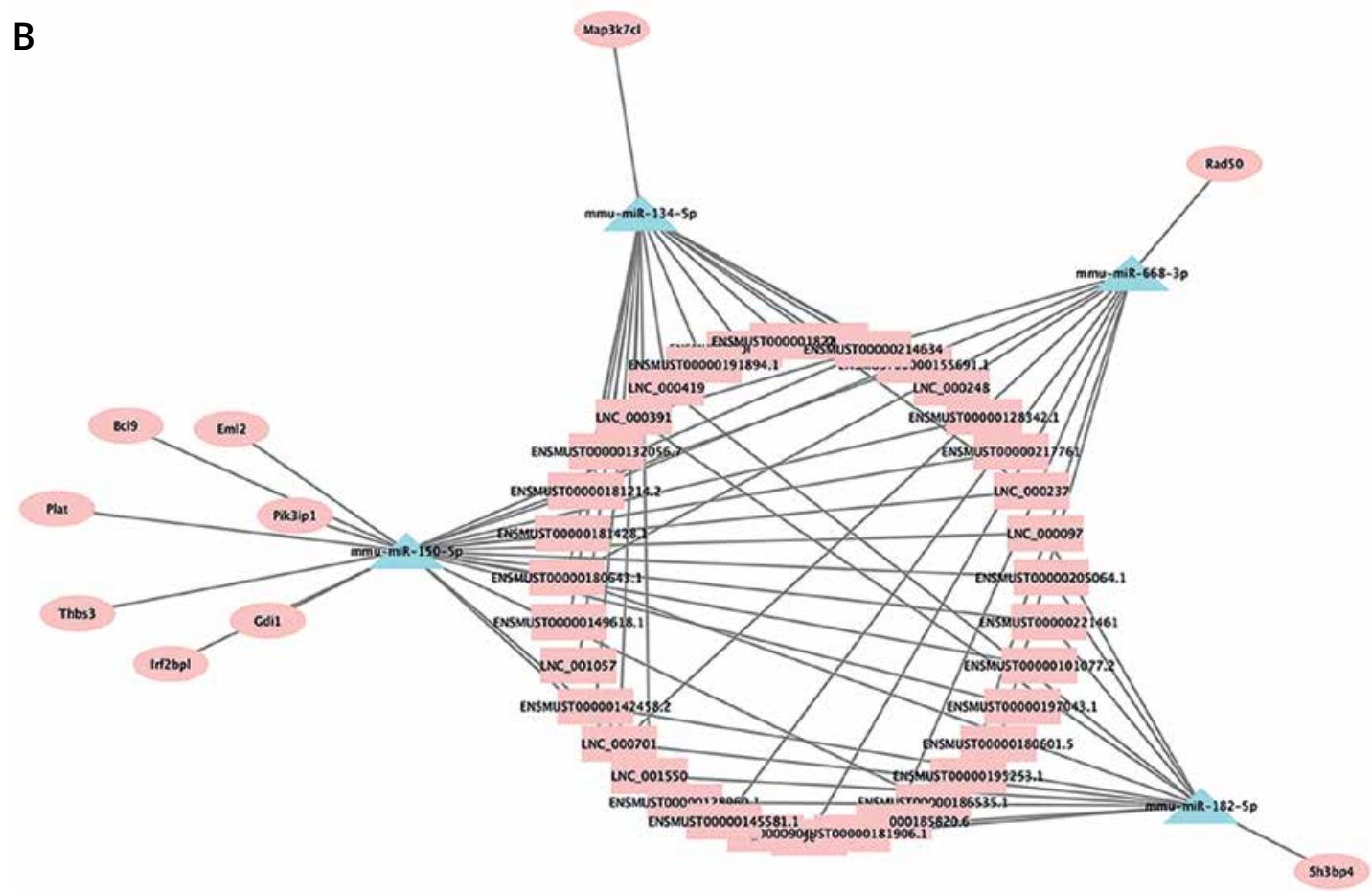

Figure 3. Cont. Predicted networks of ceRNAs. The ceRNA networks of decreased IncRNAs (A) and increased InCRNAs (B) in the ART group are depicted

A

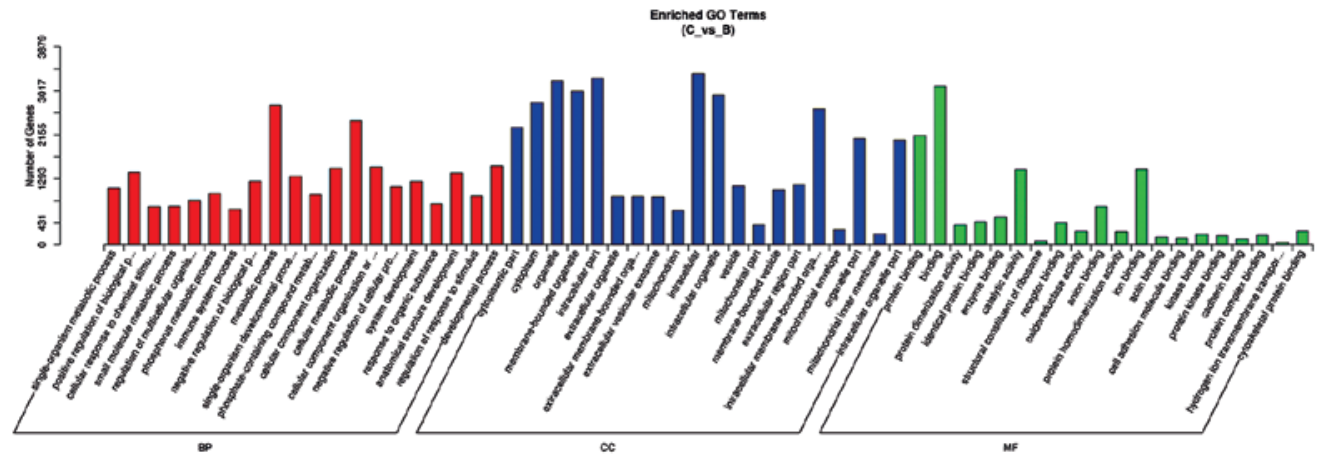

B

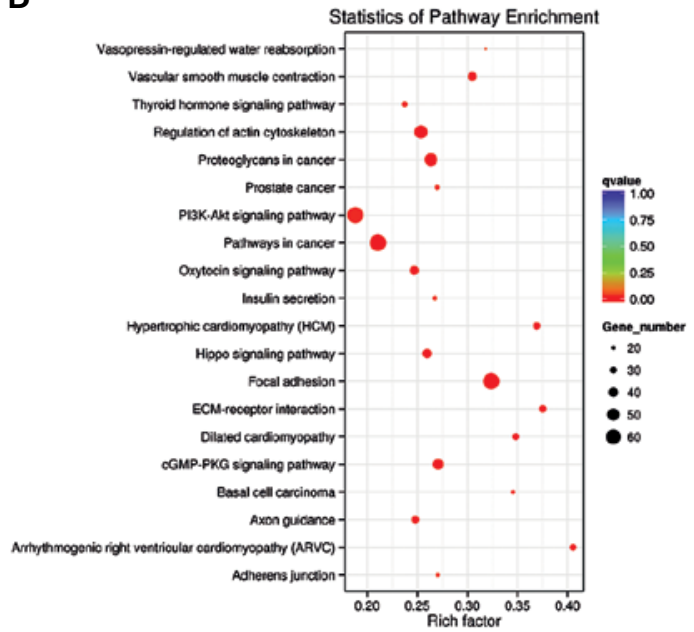

C

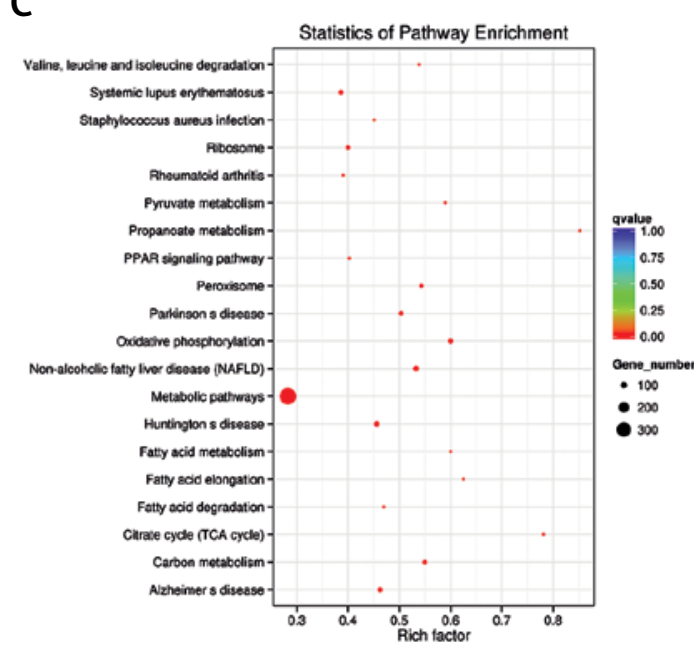

Figure 4. Go and KEGG analysis. A - GO analysis of differentially expressed mRNAs. B - KEGG pathway analysis of increased mRNAs in ART group. C - KEGG analysis of decreased mRNAs in ART group. The enrichment factor, which is the ratio of the number of differentially expressed genes in the pathway and the number of all genes annotated in the pathway, indicates the reliability of the enrichment. Q-value, which is a normalized $p$-value, indicates the significance of the enrichment 

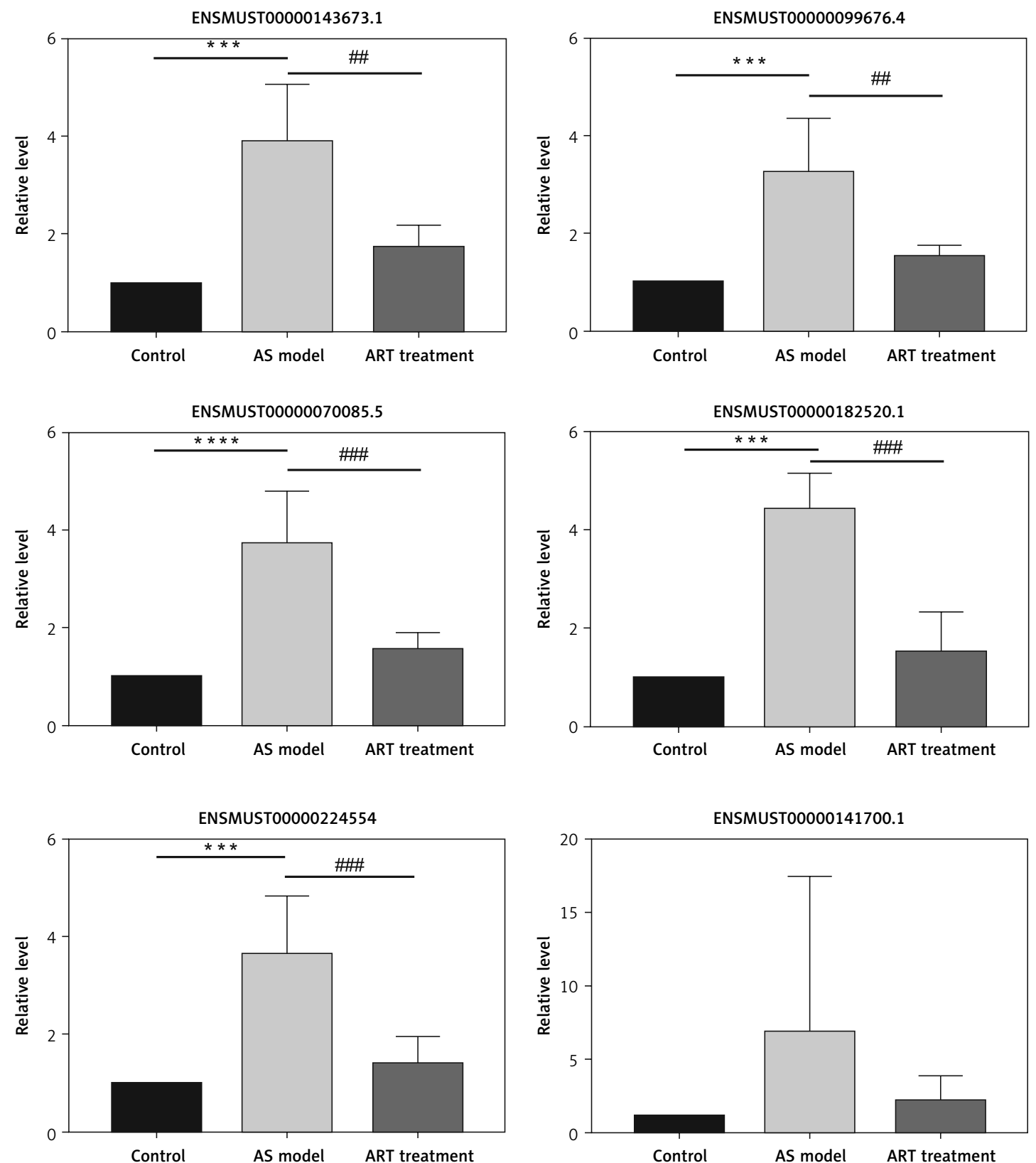

Figure 5. Validation of differentially expressed IncRNAs. The expression levels of five IncRNAs were lower in the ART model group than the atherosclerosis group ${ }^{*} p<0.01$.

ed IncRNAs in the ART group have been reported to have a protective effect on inflammation and atherosclerosis. MiR-370, which increased in the ART treatment group and might function downstream of IncRNA ENSMUST00000099676.4, has been reported to inhibit vascular inflammation [18]. The predicted downstream mRNAs regulated by miR-370 have been reported to promote the formation of atherosclerosis. For instance, nucleotide-binding oligomerization domain-containing protein 1 (Nod1) accelerates atherogenesis [19]. Deletion of $\mathrm{BH} 3$-interacting domain death agonist (Bid) inhibits cell proliferation and protects endo- thelial cells from oxidative stress related injury [20]. Interferon regulatory factor 1 (IRF1) induces VCAM-1 expression and inflammatory reactions through the activation of inducible NO synthase [21]. MiR-3473 suppressed inflammation and macrophage activation by inducing expression of IL-10 [22]. The miR-152 decreased the proliferation of HUVECs and malignant atherosclerotic progression via downregulation of Kruppel-like factor 5 (KLF5) [23]. MiR-148b, regulated by IncRNA H19, suppressed the proliferation of oxLDL-stimulated VSMCS and induced apoptosis of VSMCs [24]. Both miR-145 and miR-143 alleviated the prolif- 
eration and migration of VSMCs [25]. Let-7a attenuated ox-LDL induced endothelium injury by

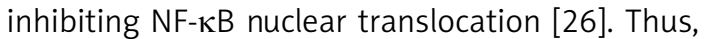
we hypothesized that ART might downregulate the formation of atherosclerosis through a ceRNA mechanism, on the basis of the decreased IncRNA in the sequencing results and the miRNAs mentioned above. Additionally, the decreased miRNAs, such as miR-150 and miR-134, predicted by the IncRNAs upregulated in the sequencing results, have been reported to enhance inflammatory reactions or atherosclerotic progression. Knockdown of miR-150 alleviated inflammatory damage by up-regulating KLF2 [27]. Prior studies have shown that miR-134, which has been reported to increase in atherosclerotic macrophages, activates lipid accumulation and the inflammatory response by regulating angiopoietin-like 4 (Angptl4) [28]. The miR-182 increased LPL expression and activates lipid accumulation in $\mathrm{APOE}^{-/-}$mice by regulating HDAC9 [29]. SH3 domain-binding protein 4 (SH3BP4), which is the predicted downstream mRNA of miR-182, was a negative regulator of amino acid-dependent mTORC1 signaling, thereby inhibiting cell growth and promoting autophagy [30]. The mRNAs and miRNAs mentioned above, which may be regulated by the IncRNAs obtained from the high-throughput sequencing results, are potential targets of ART for the treatment of atherosclerosis.

Aberrantly expressed mRNAs may reflect the functions of IncRNAs whose expression is altered by ART treatment. Thus, we conducted GO and KEGG analyses of differentially expressed mRNAs to reveal the functions of differentially expressed IncRNAs in ART treatment of atherosclerosis. GO analysis showed that differentially expressed mRNAs were linked to the functions of metabolic and immune system processes and protein binding associated with atherosclerosis, thus indicating that ART may relieve atherosclerosis by regulating these processes. The expression level of hemolytic complement ( $\mathrm{Hc}$ ), also known as $\mathrm{C} 5 \mathrm{a}$, which participates in metabolic processes and neovascularization, was downregulated in the ART treatment group [31]. Our study provides a basis for understanding the mechanism through which ART treatment mitigates atherosclerosis. KEGG analysis indicated that differentially expressed mRNAs were associated with several specific signaling pathways, such as amino acid degradation, metabolic processes, vascular smooth muscle contraction and the PI3K-Akt signaling pathway. Specific amino acids engage in the formation of macrophage foam cells. Leucine has been confirmed to have anti-atherogenic properties in vivo [32]. Downregulated mRNAs involved in degradation of valine, leucine and isoleucine in the ART-treated group may indicate elevated levels of leucine.
Although leucine levels were not measured for technical reasons, KEGG analysis suggested a possible target for ART in the treatment of atherosclerosis. In our sequencing data, LNC_000243 (chr 11: 58183820-58187387) was co-localized with 4930438A08Rik, which is associated with specific amino acid degradation. We suggest that ART may alleviate atherosclerosis by regulating the LNC_000243/4930438A08Rik/leucine axis. Upregulated $\mathrm{mRNA}$ enrichment in vascular smooth muscle contraction and the PI3K-Akt signaling pathway indicated that the ability of smooth muscle cell proliferation and migration decreased in the ART treatment group. The above enrichment analysis may potentially support future research on the mechanism through which ART decreases atherosclerosis.

In summary, our sequencing results revealed that the expression of specific mRNAs, miRNAs and IncRNAs associated with atherosclerosis was altered in the ART treatment group. The relative expression levels of IncRNAs were validated through qRT-PCR. An IncRNA-miRNA-mRNA network was constructed to explore ceRNAs involved in ART treatment of atherosclerosis. GO and KEGG analyses were used to search for possible mechanisms of ART in the treatment of atherosclerosis. Our findings suggested a possible mechanism of ART in the treatment of atherosclerosis and provided clues regarding the potential roles of IncRNAs in ART treatment. In addition, this research provides a new basis and hypothesis regarding the clinical application of ART in the treatment of atherosclerosis. There were some limitations in this study. First, we did not conduct experiments to explore the function of those RNAs and the underlying mechanism. Second, the sample size was not large enough. Further detailed research is expected to clarify the mechanism through which ART treatment decreases atherosclerosis and to identify IncRNAs involved in ART treatment, thus providing a stronger theoretical basis for the future application of ART in patients with atherosclerosis.

\section{Conflict of interest}

The authors declare no conflict of interest.

\section{References}

1. O'Neill LAJ, Kishton RJ, Rathmell J. A guide to immunometabolism for immunologists. Nat Rev Immunol 2016; 16: 553-65.

2. Tuñón J, Badimón L, Bochaton-Piallat M-L, et al. Identifying the anti-inflammatory response to lipid lowering therapy: a position paper from the working group on atherosclerosis and vascular biology of the European Society of Cardiology. Cardiovasc Res 2019; 115: 10-9.

3. Libby P. Inflammation in atherosclerosis. Nature 2002; 420: 868-74. 
4. Ketelhuth DFJ, Lutgens E, Bäck M, et al. Immunometabolism and atherosclerosis: perspectives and clinical significance: a position paper from the Working Group on Atherosclerosis and Vascular Biology of the European Society of Cardiology. Cardiovasc Res 2019; 115: 1385-92.

5. Eken MK, Ersoy GS, Kaygusuz El, et al. Etanercept protects ovarian reserve against ischemia/reperfusion injury in a rat model. Arch Med Sci 2019; 15: 1104-12.

6. Lu D, Peng F, Li J, et al. Urotensin II promotes secretion of LTB4 through 5-lipoxygenase via the UT-ROS-Akt pathway in RAW264.7 macrophages. Arch Med Sci 2019; 15: 1065-72.

7. White NJ. Qinghaosu (artemisinin): the price of success. Science 2008; 320: 330-4

8. Kumari A, Karnatak M, Singh D, et al. Current scenario of artemisinin and its analogues for antimalarial activity. Eur J Med Chem 2019; 163: 804-29.

9. Shi C, Li H, Yang Y, Hou L. Anti-inflammatory and immunoregulatory functions of artemisinin and its derivatives. Mediators Inflamm 2015; 2015: 435713.

10. Jiang Y, Du H, Liu X, Fu X, Li X, Cao Q. Artemisinin alleviates atherosclerotic lesion by reducing macrophage inflammation via regulation of AMPK/NF- $\mathrm{KB} / \mathrm{NLRP3}$ inflammasomes pathway. J Drug Target 2020; 28: 70-9.

11. Cao Q, Jiang Y, Shi J, et al. Artemisinin inhibits the proliferation, migration, and inflammatory reaction induced by tumor necrosis factor- $\alpha$ in vascular smooth muscle cells through nuclear factor kappa B pathway. J Surg Res 2015; 194: 667-78.

12. Jedynak M, Siemiątkowski A, Milewski R, Mroczko B, Szmitkowski M. Diagnostic effectiveness of soluble triggering receptor expressed on myeloid cells-1 in sepsis, severe sepsis and septic shock. Arch Med Sci 2019; 15: 713-21.

13. Marchese FP, Raimondi I, Huarte M. The multidimensional mechanisms of long noncoding RNA function. Genome Biol 2017; 18: 206.

14. Liu Y, Zheng L, Wang Q, Hu Y-W. Emerging roles and mechanisms of long noncoding RNAs in atherosclerosis. Int J Cardiol 2017; 228: 570-82.

15. Du H, Zhao Q, Zang H, Chang C, Li X. Artemisinin attenuates the development of atherosclerotic lesions by the regulation of vascular smooth muscle cell phenotype switching. Life Sci 2019; 237: 116943.

16. Poredoš P, Spirkoska A, Ježovnik MK. In patients with superficial vein thrombosis the inflammatory response is increased and related to the recanalization rate. Arch Med Sci 2019; 15: 393-401.

17. Cao L, Zhang Z, Li Y, Zhao P, Chen Y. LncRNA H19/miRlet-7 axis participates in the regulation of ox-LDL-induced endothelial cell injury via targeting periostin. Int Immunopharmacol 2019; 72: 496-503.

18. Tian D, Sha Y, Lu J-M, Du X-J. MiR-370 inhibits vascular inflammation and oxidative stress triggered by oxidized low-density lipoprotein through targeting TLR4. J Cell Biochem 2018; 119: 6231-7.

19. Kanno S, Nishio H, Tanaka T, et al. Activation of an innate immune receptor, Nod1, accelerates atherogenesis in Apoe ${ }^{-/}$mice. J Immunol 2015; 194: 773-80.

20. Bai L, Ni H-M, Chen X, DiFrancesca D, Yin X-M. Deletion of bid impedes cell proliferation and hepatic carcinogenesis. Am J Pathol 2005; 166: 1523-32

21. Warfel JM, D’Agnillo F. Anthrax lethal toxin enhances TNF-induced endothelial VCAM-1 expression via an IFN regulatory factor-1-dependent mechanism. J Immunol 2008; 180: 7516-24.
22. Wu C, Xue Y, Wang P, et al. IFN- $\gamma$ primes macrophage activation by increasing phosphatase and tensin homolog via downregulation of miR-3473b. J Immunol 2014 193: 3036-44

23. Wang W, Zhang Y, Wang L, et al. MircroRNA-152 prevents the malignant progression of atherosclerosis via down-regulation of KLF5. Biomed Pharmacother 2019; 109: 2409-14

24. Zhang L, Cheng H, Yue Y, Li S, Zhang D, He R. H19 knock down suppresses proliferation and induces apoptosis by regulating miR-148b/WNT/ $\beta$-catenin in ox-LDL -stimulated vascular smooth muscle cells. J Biomed Sci 2018 25: 11.

25. Boettger T, Beetz N, Kostin S, et al. Acquisition of the contractile phenotype by murine arterial smooth mus cle cells depends on the Mir143/145 gene cluster. J Clin Invest 2009; 119: 2634-47.

26. Bao M, Zhang Y, Lou X, Cheng Y, Zhou H. Protective effects of let-7a and let-7b on oxidized low-density lipoprotein induced endothelial cell injuries. PLoS One 2014; 9: e106540.

27. Yang X, Zhang Q, Gao Z, Yu C, Zhang L. Down-regulation of MiR-150 alleviates inflammatory injury induced by interleukin 1 via targeting Kruppel-like factor 2 in human chondrogenic cells. Cell Physiol Biochem 2018; 47: 2579-88.

28. Lan G, Xie W, Li L, et al. MicroRNA-134 actives lipoprotein lipase-mediated lipid accumulation and inflammatory response by targeting angiopoietin-like 4 in THP-1 macrophages. Biochem Biophys Res Commun 2016; 472: 410-7.

29. Cheng H-P, Gong D, Zhao Z-W, et al. MicroRNA-182 promotes lipoprotein lipase expression and atherogenesisby targeting histone deacetylase 9 in apolipoprotein e-knockout mice. Circ J 2018; 82: 28-38.

30. Kim Y-M, Stone M, Hwang TH, et al. SH3BP4 is a negative regulator of amino acid-Rag GTPase-mTORC1 signaling. Mol Cell 2012; 46: 833-46.

31. Nozaki M, Raisler BJ, Sakurai E, et al. Drusen complement components $\mathrm{C} 3 \mathrm{a}$ and $\mathrm{C} 5 \mathrm{a}$ promote choroidal neovascularization. Proc Natl Acad Sci USA 2006; 103 2328-33.

32. Grajeda-Iglesias C, Aviram M. Specific amino acids affect cardiovascular diseases and atherogenesis via protection against macrophage foam cell formation: review article. Rambam Maimonides Med J 2018; 9: e0022. 